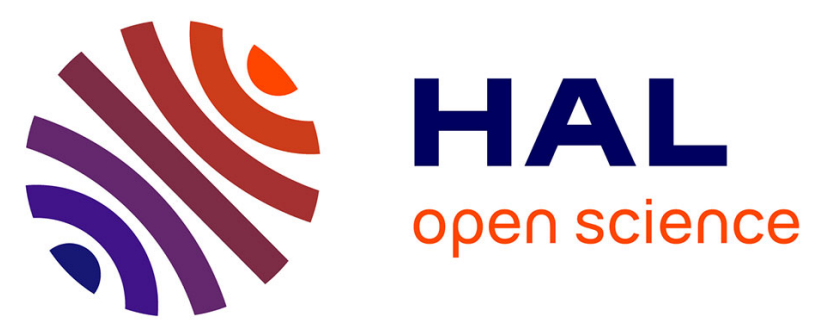

\title{
How effective is to look at a vehicular network under a social perception?
}

Felipe Domingos da Cunha, Aline Carneiro Viana, Raquel A. F. Mini, Antonio A. F. Loureiro

\section{- To cite this version:}

Felipe Domingos da Cunha, Aline Carneiro Viana, Raquel A. F. Mini, Antonio A. F. Loureiro. How effective is to look at a vehicular network under a social perception?. Wireless and Mobile Computing, Networking and Communications (WiMob), 2013 IEEE 9th International Conference on, Oct 2013, Lyon, France. pp.154 - 159, 10.1109/WiMOB.2013.6673355 . hal-00926339

\section{HAL Id: hal-00926339 \\ https://hal.inria.fr/hal-00926339}

Submitted on 13 Jan 2014

HAL is a multi-disciplinary open access archive for the deposit and dissemination of scientific research documents, whether they are published or not. The documents may come from teaching and research institutions in France or abroad, or from public or private research centers.
L'archive ouverte pluridisciplinaire HAL, est destinée au dépôt et à la diffusion de documents scientifiques de niveau recherche, publiés ou non, émanant des établissements d'enseignement et de recherche français ou étrangers, des laboratoires publics ou privés. 


\title{
How effective is to look at a Vehicular Network under a Social Perception?
}

\author{
Felipe D. Cunha*, Aline Carneiro Vianna ${ }^{\ddagger}$, Raquel A. F. Mini ${ }^{\dagger}$, Antonio A.F. Loureiro* \\ *Federal University of Minas Gerais, Brazil - \{fdcunha, loureiro\}@dcc.ufmg.br \\ ${ }^{\dagger}$ Pontifical Catholic University of Minas Gerais, Brazil - raquelmini@pucminas.br \\ ${ }^{\ddagger}$ HIPERCOM Research Team - INRIA - aline.viana@inria.fr
}

\begin{abstract}
Vehicular Mobility is strongly influenced by the speed limits and direction of the public roads. At the same time, the driver's behavior produces great influences in vehicular mobility. People tend to go to the same places, at the same day period, through the same trajectories, which le ad them to the appearance of driver's daily routines. These routines lead us to the study of mobility in VANETs under a social perspective and to investigate how effective is to explore social interactions in this kind of network. The work herein proposed presents the characterization and evaluation of a realistic vehicular trace found in literature. Our aim is to study the vehicles' mobility in accordance to social behaviors. With our analysis is possible to verify the existence of regularity and common interests among the drivers in vehicular networks. Finally, we discuss how the social metrics may be used to improve the performance of protocols and services in Vehicular Networks.
\end{abstract}

\section{INTRODUCTION}

Vehicular Ad hoc Networks (VANETs) are a special type of Ad hoc networks formed by vehicles with processing and wireless communication ability, traveling on streets or highways. Commonly, the vehicles can communicate directly or by the use of a roadside unit. Through this structure, vehicles can access network services and obtain data from other networks, such as Internet. Due to this nature, VANETs can be established in different environments such as in urban centers and highways [1], [3].

VANETs are proposed to assist the drivers in the transit, to avoid collisions, to provide entertainment to drivers and passengers, and to contribute to the creation of an intelligent traffic system. An important application of this type of network is to alert the driver about road conditions and imminent crashes. Recent studies show that $60 \%$ of car crashes could be avoided if the drivers were warned some seconds before the collision [3]. In this context, the interaction among vehicles can strongly contribute to broadcast an alert message and reduce the crash ratio [11].

The communication in VANETs is influenced by mobility patterns of the vehicles: they move and stop according to the speed limits and directions imposed by the public roads. Furthermore, the day period can influence the vehicles mobility. In rush hours, the traffic can be slower and traffic jams can force the driver to stop the car or to change the route. This is a scenario where the vehicles density will be higher. In other hand, weekends and early mornings are considered quiet traffic scenarios, with low vehicles density. This density variation in different hours reinforces the characteristic of dynamic topology of VANETs, making the communication a challenging task in some scenarios.

The mobility is also influenced by the behavior of the driver and its routine. At the weekends, destinations like camping, malls, churches are chosen to leisure and entertainment, and are frequently visited. On the contrary, on weekdays people tend to repeat their paths at the same time and to the same destination, such as: school, work, university, restaurant, etc. In this context, it is likely to find vehicles sharing the same destination and having similar behavior, which suggests studying the mobility in VANET under a social network perspective.

In his paper entitled Small-World Problem [7], Milgram presents the concept of social networks, showing that people with similar interests tend to relate among themselves. Other important concept is that there are people responsible for connecting groups of people that have a large degree of interaction in the network. In this work, we present a study of the social metrics applied in VANETs. Although similar evaluations can be found in literature [4], [6], [5], our work differs from these by the fact that social metrics are used to characterize vehicles' mobility in realistic dataset. We also discuss how these metrics can be applied to improve the performance of some services or existing infrastructures in VANETs. In this context, we focus on the investigation of the following question: How effective is to look to a vehicular network under a social perception, considering the social behavior of the drivers? Through numerical analysis, it is possible to identify peculiar social characteristics in VANETs.

This paper is organized as follows. In Section II, we present a brief survey of the relevant related work. In Section III, we define the dataset used in the analysis, the metrics and the main assumptions of the modeling. In Section IV, we present the quantitative results according to each evaluated metric. In Section $\mathrm{V}$, we discuss the perspective of applying the evaluated metrics on the design and evaluation of the VANETs protocols. Finally, in Section VI, we present the final conclusions of this work and future works.

\section{BACKGROUND}

In this Section, we survey available traces describing vehicular mobility as well as related works found in literature.

\section{A. Vehicular Traces}

Vehicular traces are a special dataset type that contains information about the trajectory of vehicles. Such traces are 
attractive since they exhibit the real behavior of vehicles in a certain scenario. In literature, there are real and realistic traces. The real traces contain information collected from a group of vehicles, with the use of some localization system device (e.g. GPS). The realistic traces are created by the junction of maps of particular locality, traffic information of this locality, and a mobility' simulator. In this section, we describe traces commonly used to validate protocols.

San Francisco: This real trace describing mobility of taxi cabs in San Francisco [10]. Each taxi has a GPS receiver, and the trace contains GPS coordinates of 500 taxis, collected over 30 days, from May 17 to June 10 of 2008.

Zurich: A multi-agent traffic simulator, developed by ETH Zurich, created a realistic trace for the city of Zurich, Switzerland [8]. This trace contains public and private traffic with high levels of realism, reproducing the behavior of a large city during 24 hours. With 25, 000, 000 records (direction/speed) of 260,000 vehicles, the trace covers an area of $250 \mathrm{~km} \times 260 \mathrm{~km}$.

Shanghai: The SUVnet-trace is a real trace that has the GPS information from more than 4,000 taxis in the Shanghai city [12]. This taxi group has been observed during 28 days, from January 31 to February 27 of 2007.

Cologne: The TAPASCologne Project is a realistic trace that reproduces with the highest realism the car traffic in the Cologne city, in Germany [2]. The process to make this trace includes important tools of the literature like: OpenStreetMap, SUMO simulator mobility, the information of traffic demand on the city. It brings information of 700, 000 records of individual car trips, during 24 hours in an area of $400 \mathrm{~km}^{2}$.

Luxembourg: This is a realistic trace that presents commuting traffic over the area of Luxembourg [9]. It has been generated by the VehiLux model and augmented with Gawron's dynamic route assignment algorithm. The trace cover an area of $51 \mathrm{~km} \times 44 \mathrm{~km}$ during 11 hours and has 110,188 records.

\section{B. Related Work}

The concepts of Social Networks have been explored in different research areas, mainly to understand the human mobility. In this context, the focus is to analyze physical encounters of the nodes in order to understand the network topology and to improve the performance of the network protocols. Moreover, in VANETs, social analysis can be used to assist the design of protocols, aiming the provision of better services to vehicles. Furthermore, it is important to observe the evolution of the traffic in specific areas and over the time, in order to improve protocols and services performance in VANETs.

In [4], Fiore et al. present a deep analysis of the topological properties of VANETs. The authors use social metrics to investigate the temporal evolution of network topology. Results show how it is possible to take advantage of the vehicular mobility to improve the performance of network protocols. This work is based only in analytical mobility models.

In the same context, in [6], the authors discuss how social metrics can be employed to improve the performance of the routing protocols in VANETs. The analysis, however, is based on a short period of only 2 hours. We argue that short observation period is not enough to extract social behavior in vehicular mobility.

Other social metrics investigation is presented in [5]. This work discusses several universal laws of social networks and presents numerical results of the real traces' evaluation. The authors have used two publics dataset San Francisco and Shanghai, describing taxis movements. The social metrics are estimated, and they show the existence of social routine in the VANETs, including the power-law distribution and small world phenomenon. This work however, only considers traces describing mobility of cabs, which has random trajectories with low probability to have routines or similar behaviors.

In [13], it is presented a macro and microscopic evaluation of the Cologne trace. The macroscopic analysis involves the evolution of vehicular density and the dynamics of large-scale flows of vehicles through the metropolitan region. In addition, the microscopic analysis considers the distribution of vehicles in the area and the encounters among them. Through the performed evaluation, it is possible to observe the evolution of the traffic over the region and period. Also, the authors show the importance of considering the dynamics of the road traffic in the design of protocols and services to VANETs.

\section{Methodology}

This section details the studied trace, the modeling used to create the social graph and the evaluated metrics.

\section{A. Trace Characterization}

We decided to use the Zurich Trace [8]. This choice is due to two factors: (i) it represents a trace with records of personal vehicles describing people routines in an urban area; Traces such as San Francisco and Shanghai describe taxis decisions that are strongly dependent on the decisions of passengers that are randomly collected on the streets; (ii) the $24 \mathrm{~h}$ of records are freely available, contrarily to $2 \mathrm{~h}$ of the Cologne trace and the $11 \mathrm{~h}$ of the Luxembourg trace. The Zurich trace represents the evolution of the urban traffic along the day, including the variation like rush hours and daybreaks. We can see this evolution in Figures 1(a) and 1(b). In these figures, it is possible to observe two intervals time that have the major amount of vehicles, called rush hours: $6-9$ am and $2-5 \mathrm{pm}$.

The average speed is $25 \mathrm{~km} / \mathrm{h}$ and in Figure 1(a) we can see this variation. The minimum speed is around $1 \mathrm{~km} / \mathrm{h}$, which represents the presence of traffic jam in some places. Moreover, the maximum speed remains constant, around $30 \mathrm{~km} / \mathrm{h}$. This value is attributed to the vehicles far from downtown, which travel through streets with low traffic.

The whole trace has movement information of 260,000 vehicles, and in Figure 1(b), we can observe the distribution of these vehicles along the day. It is possible to see a concentration of vehicles in rush hours: 173,470 vehicles at $7 \mathrm{am}$, and 132,633 vehicles at $3 \mathrm{pm}$. When we compare Figures 1(a) and 1(b) in rush hours, it is possible to perceive a decrease in speed. This happens due to the high number of vehicles in the streets and highways, leading the drivers to reduce the speed or to stop the vehicles. 


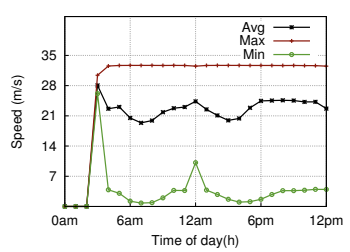

(a) Daily Speed.

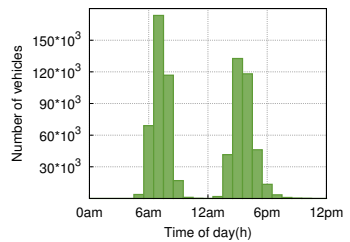

(b) Daily Traffic.
Fig. 1. Daily evolution of the Zurich trace.

We show in Figures 2(a-d) the heat map of the traffic evolution in the area. We choose to use the traffic from 6 to $7 \mathrm{am}$, and from to $2 \mathrm{pm}$ to $3 \mathrm{pm}$, in order to demonstrate the vehicles distribution over the city. In these figures, the black color represents the area with high vehicles density, and the yellow color represents the area with low vehicles density. As can be seen, the streets with black color in the center of figure represent the high traffic density at the center of Zurich. In addition, away from the center, the yellow color represents the low density streets. When we compare Figures 2(a) and 2(b), we observe the increase of traffic in the center. We also note the same behavior with Figures 2(c) and 2(d), which corresponds to the evolution of traffic presented in the Figure 1(b).

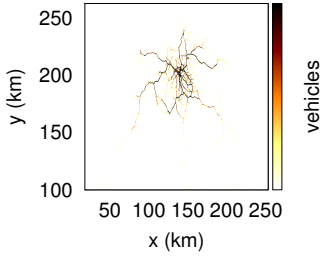

(a) $6 \mathrm{am}$.

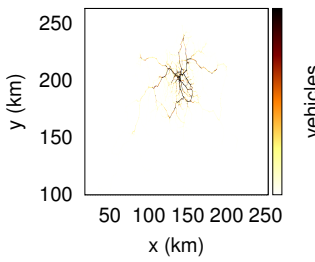

(c) $2 \mathrm{pm}$.

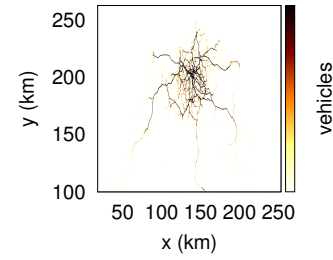

(b) $7 \mathrm{am}$.

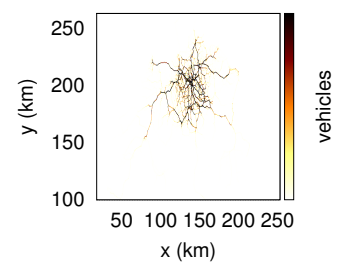

(d) $3 \mathrm{pm}$.
Fig. 2. Heat map of traffic evolution in rush hours.

\section{B. Modeling}

The model considers the encounter among vehicles, i.e., when two vehicles are within communication range of each other. In VANETs, the vehicle-to-vehicle communication might be explored in order to increase the probability of the message to reach the destination and consequently, to increase the delivery ratio. Thus, the better understanding of the evolution of these encounters can help us to improve the network communication.

In our evaluation, we consider communication range of $100 \mathrm{~m}$ among vehicles, according to the protocol 802.11p. The Zurich trace describes mobility in urban areas and highways, where the average speed is $25 \mathrm{~km} / \mathrm{h}$. Therefore, according to this speed and the communication range, we consider the minimum contact time of one encounter equal 15 seconds(s). Thus, if two vehicles are inside each others communication range, at least for $15 \mathrm{~s}$, we consider that an encounter happened.

Then, we map vehicles mobility and encounters described in the trace into a temporal graph. We first divide the whole trace into discrete time slots of duration $t=1 \mathrm{~h}$. Since vehicle traces usually describe fast changes in the topology, short time slots are recommended to better capture traffic changes. In addition, traffic peaks are usually assumed to happen in different hours of the day.

The graph at the time $t$ is an undirected and can be formally defined as a graph $G(t)=(V, E)$, where $V$ represents the set with all vehicles $v_{i}$ and $E$ represents the set of edges $e_{i j}$. In the temporal graph $G(t)$, an edge $e_{i j}(t)$ exists between the vehicle $v_{i}$ and $v_{j}$ during time $t$, with $i \neq j$. All metrics are evaluated hourly, considering the temporal graph $G(t)$. Considering the fact that the Zurich trace describes $24 \mathrm{~h}$ of vehicle mobility, 24 graphs are generated, describing the vehicles encounters in time windows of $1 \mathrm{~h}$.

\section{Metrics Evaluated}

Below the social metrics chosen for this evaluation will be described considering the aspects and characteristics of the mobility of vehicles. As for vehicles mobility, we classify these metrics in macroscopic and microscopic metrics [13]. The macroscopic metrics represent measures of the network global state, which can portray the general behavior of all vehicles and the evolution of the temporal graph. The microscopic metrics define individual values for the vehicle representing the behavior of a unique vehicle. We select as macroscopic metrics the Distance, the Density and the Edge Persistence, and as microscopic metrics, we select the Node Degree, the Cluster Coefficient, and the Closeness Centrality.

\section{Macroscopic Metrics:}

Distance: this is a metric that comprises the length of a path between a pair of vehicles in terms of the number of hops. In VANETs, the distance can represent the existence of common interest among the drivers. If the distance between the vehicles $v_{i}$ and $v_{j}$ is high, it implies that they are physically separated, and probably they not visit nearby places. Otherwise, if the distance is small, $v_{i}$ and $v_{j}$ may tend to visit close places or have congruent trajectories. Generally, graphs with small world behavior have small distances.

Density: Represents how dense is the network, i.e., the amount of connections existent between the vehicles. In the context of VANETs, urban regions can have higher densities than rural areas. This is particularly correct in downtown region, where the traffic is slow and there are traffic jams. This metric compute the ratio between the number of existing edges $e(t)$ in the graph and the number of edges of a complete graph $|V||V-1| / 2$.

Edge persistence: is a metric that represents the persistence of an encounter among two vehicles. In this analysis, we consider the edge persistence as the number of times the 
vehicles $v_{i}$ and $v_{j}$ encounter over the time. We compute this metric in function of the edge weight, which represents the number of times that this encounter $e_{i j}(t)$ happens.

\section{Microscopic Metrics}

Node Degree: determines the number of distinct encounters that a vehicle has during a period. This metric can be influenced by the trajectory and the period of the day. If a vehicle passes through a region with higher density traffic or in a rush hour, its degree tends to be high. In other hand, in regions far from the downtown or in hours with low traffic, its degree can be low or null. As a formal definition, a vehicle $v_{i}$ at time $t$ is defined by: $\operatorname{Degree}_{i}(t)=\left\|\left\{v_{j} \mid \exists e_{i j}(t)\right\}\right\|$.

Cluster Coefficient: this is a metric that evaluates how close are the neighbors of a vehicle $v_{i}$ in the graph, i.e., the probability that two neighbors of $v_{i}$ have already met. This metric can represent regions with traffic jam, describing a high cluster coefficient. Besides, if the network presents the small world phenomenon, its cluster coefficient will also be elevated [14].

Closeness Centrality: This metric measures the centrality of the vehicle according to its distance to the others vehicles in the graph. The more central a vehicle is the lower is its total distance to all others vehicles. Despite being a global metric, its value can also show how central is the trajectory of a vehicle. Likely, a vehicle that travels only in downtown tends to have a low closeness centrality.

\section{NumericAl Results}

In this section, we focus on the analysis of the metrics discussed in the Section III. All results are computed using the temporal graph $G(t)$, when $t=1 h$.

\section{A. Distance}

It is worth to mentioning this distance metric considers the number of edges that are between two nodes, without ponder their edge weight. In VANETs, this is a metric that can represents if two vehicles have visited common locations in close time, i.e., vehicles with the similar routine tend to have little distance. On the other hand, vehicles that travel in different times, or through distant regions tend to have big distances. The graphic in the Figure 3(a) shows the average of the graphs distance over time. It is possible to observe the behavior of the distance when the number of vehicles increases in network.

In the period where there is no traffic, the value of the distance is represented as 0 , and the distance is not considered. From $4 \mathrm{am}$, the distance increases with the growth of the number of vehicles. However, it is possible to note that the measured distances remain short despite of their increase when we compare to the number of vehicles circulating on the streets. For instance, the measured average distance at $7 \mathrm{am}$ is of 30 hops, while the number of vehicles is 173,470 . Other important point is that there is a similarity in the distance when we compare the two rush hours. In both hours, the distance increases with the traffic intensify. This happens because, when the number of vehicles in the street increases, there is a tendency to form queues of cars, and consequently increase the distance in the graph. Considering the number of vehicles and the length of distances, it is possible to conclude that this graph presents a small world behavior (i.e. great number of nodes and short distances present in social networks).

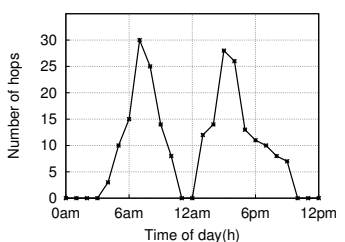

(a) Distance.

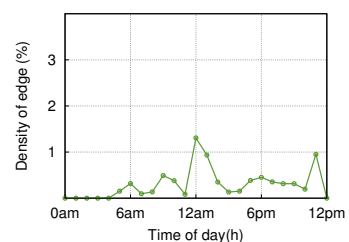

(b) Edge Density
Fig. 3. Daily evolution of macroscopic metrics.

\section{B. Density}

In this analysis, we evaluated the global density of the graph. Figure 3(b) shows the evolution of the network density over time. It is possible to observe that the evolution of the density does not exceed the value of $2 \%$. Despite of the significantly traffic increase during rush hours, the density remains lower. This happens because the streets have direction restrictions and speed limits, which define the vehicles trajectory and the set of vehicles to possible encounters. Other factor that contributes to the low density is the traffic jam, which reduces the speed of the vehicles and prevents new encounters. In this situation, the vehicle tends to keep the same neighbors while it passes in a specific region. Furthermore, the density evolution shows that the limits imposed by public roads have an influence in the encounter probability of vehicles.

\section{Edge Persistence}

Persistence encounters happen due to drivers sharing similar routines, e.g. pass through the same path to go to work or back to home at the same hours. Hence, edge persistence may be used to identify mobility similarity among vehicles. We measure the edge persistence of vehicles at the two identified rush periods of the day, due to their higher number of vehicles and encounters. The repeated encounters percentage is then computed based on the comparison of the number of encounters happened among the periods $6-9 \mathrm{am}$ and $2-5 \mathrm{pm}$.

In the former period, 376,245 encounters happened while 338,513 encounters happened in the latter period. The edge persistence for this analysis is $43 \%$ of the edges, indicating that a high amount of vehicles tend to have similar mobility in the city, with similar routines. Besides the restrictions imposed by streets, traffic lights, and speed limits, the path decisions of the drivers have a strong influence in the definition of their trip time and trajectory.

\section{Node Degree}

As previously discussed the traffic density in a metropolitan area varies over the day. In Figure 5(a), we can observe this behavior. The rush hours present an increase in the max, average and median degree of the vehicles. At 7am, the graph presents the highest degree value of 1,355 , which may describe a vehicle that only crosses the streets with high traffic density and makes 
a big trip. A high degree value can be also seen at $4 \mathrm{pm}$. However, when we look at the average value, at least $50 \%$ of vehicles present an increased, due to the traffic daily evolution. This is an acceptable behavior, because with the increase of vehicles it is expected a greater amount of encounters.

Many social networks have been considered as scalefree networks, i.e., networks that have a degree distribution following a power-law behavior. In Figures 4(a-d), we present the degree distribution in rush hours. In these hours, we have approximately 100, 000 vehicles in each period. The curves in the graphs show that a power law describes well the degree distribution, which suggests that the Zurich graph is scale-free. We can see that few vehicles have a high degree, and the major amount of vehicles have a low degree, with values close to $10^{2}$.

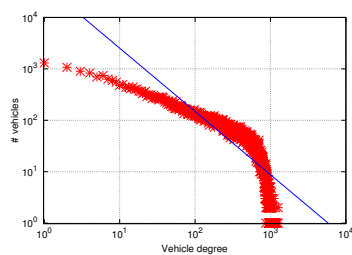

(a) 6 am.

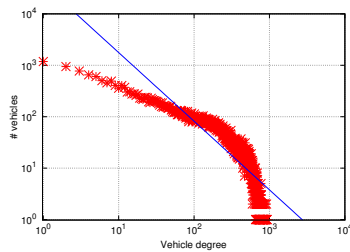

(c) $2 \mathrm{pm}$.

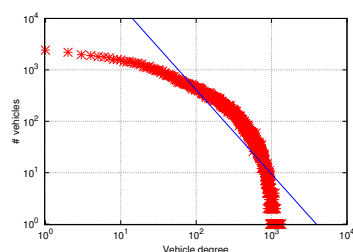

(b) $7 \mathrm{am}$.

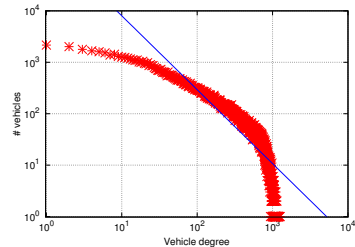

(d) $3 \mathrm{pm}$.
Fig. 4. Vehicle degree evolution during the day.

\section{E. Cluster Coefficient}

The cluster coefficient is a very important metric in social networks, since it represents the organization of the graph and how close are the neighbors of a node in the graph. In the context of a VANET, the cluster coefficient can aggregate the vehicles that go to the same direction, that have the same interests, or that pass through the same place. Figure 5(b) presents the max, average, and median value of the cluster coefficient for temporal graph $G(t)$ during a day. It is possible to observe that the max cluster coefficient is 1 in rush hours, showing that there are vehicles that all of their neighbors are connected. In metropolitan areas, traffic jams and intersection streets favor the clusters appearance, composed of vehicles sharing the same road and traffic conditions.

When we look at the average and median cluster coefficient values, we observe their increase during the rush hours. In particular, at $7 \mathrm{am}$ and at $4 \mathrm{pm}$ the average cluster coefficient is 0.5 . This represents in average, $50 \%$ of the vehicle's neighbors had an encounter over the time. It is interesting to note that a network with a small world behavior has a high value of cluster coefficient, and the Zurich trace tends to perform as a small world network when the density of vehicles is high. This

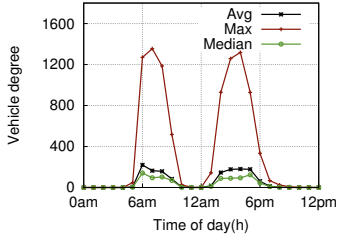

(a) Node Degree.

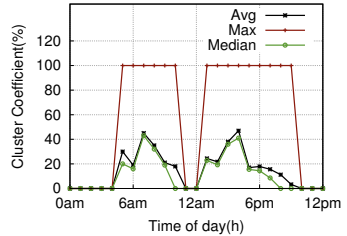

(b) Cluster Coefficient.

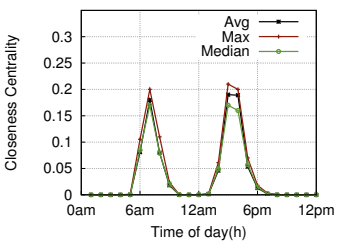

(c) Closeness Centrality.

Fig. 5. Daily evolution of microscopic metrics.

is due to the fact that with more vehicles, more encounters happen and more groups are formed. In addition, traffic jams contribute to form vehicles groups that have common junction points in their trajectories. Finally, it is worth to note the proximity between the average and the median values of the cluster coefficient, which is a good indication that the average is very representative value of the network cluster coefficient.

\section{F. Closeness Centrality}

The evaluation of the closeness centrality in a graph is important to understand how close are the nodes. In VANETs, this metric can quantify the proximity between the vehicles, and consequently better understand the mobility pattern. The Figure 5(c) present the max, average and median values of the closeness centrality during a day.

By definition, the higher the value of the closeness of a node, the lower is relative distance to other nodes. Thus, in the graph, we can observe that the curve of closeness increases with the increase of vehicles number. This is due to the fact that with more nodes in the graph, there is a tendency to create more shortcuts between the nodes, and the distance tends to decrease. We can also observe that the maximum closeness value is close to the average and median. As the closeness considers the distance to all nodes, with a high number of nodes, this value tends to be low. This happens mainly in cases where we have many nodes with low degree. On the other hand, in periods of low traffic the value of closeness is very low, which indicates that the graph does not have a central vehicle.

\section{Social Properties Applied}

In this work, we have analyzed a vehicular trace under a social perspective, aiming to characterize it according to metrics that lead us to comprehend the vehicular mobility. It is possible to make use of these metrics to improve the network performance of communication protocols and services, aiming to help drivers on their trip. In the following, we will discuss scenarios where the previously analyzed metrics can be used to improve the VANETs performance. 
Infrastructure: The communication between vehicles can happen in three modes: vehicle-to-vehicle, vehicle-to-infrastructure, and in hybrid way, i.e., by combining the two others modes. In this context, determine the best locations to deploy roadside units, with the goal of providing continuous connectivity to drivers during their trajectories becomes a challenge. In macroscopic metrics, the information about the vehicles flows in the network can help in this task. For instance, the density metric that measures the number of encounters over time can be used together with the physical position of vehicles to assist in the deployment of new roadsides units, in order to maximize the network coverage. Using this metric, it is possible to detect places demanding high number of connections, which can require more roadside units installed, guarantee a good Internet access.

Network Protocols: Many are the challenges for the communication protocols in this type of network, such as the variation in density and mobility. Thus, providing secure communication and message delivery becomes a challenging task in many scenarios. An important issue for communication is to ensure message delivery in situations where the connection time between vehicles is minimal, and it is necessary to reach the largest number of vehicles as possible. In this case, making use of information provided by microscopic metrics can help deliver the message more efficiently. An example is the use of metric node degree, which describes the number of neighbors of a vehicle. Thus, choosing a vehicle more popular, i.e., with a larger number of neighbors, increases the chance of delivering the message to a wider group. Another important microscopic metric is the closeness centrality, which describes how central is the node in terms of the proximity to other nodes. The choice of central nodes to start the dissemination of information can ensure a wider delivery of the message. The metric density can also be used to promote the routing in the network, avoiding places with low density or disconnected.

Vehicular Applications: Applications in VANETs are proposed in order to assist the driver and guarantee a safe direction. In this scenario, various types of applications can be offered as security alerts, travel assistance in roads, and entertainment. However, every application has the requirement to ensure good performance. Thus, microscopic and macroscopic metrics can be used in different contexts. For instance, the metric edge persistence can be used to identify the drivers who have the same routine and enable resource sharing among them. In other scenarios, the metric cluster coefficient may be used to target a specific group of users, who have a common interest, such as publicity, alert messages and sharing resources. Vehicles belonging to the same group tend to have interests in common, or be physically close. Finally, drivers habitually use different location systems to find places or to create paths to an informed destination. However, such paths are usually built without considering the live information of the vehicles traffic. In this scenario, it is possible to use the metrics density of a region or the node degree of the vehicles, to infer traffic conditions, improving the suggested route.

\section{CONCLUSION}

In this work, we presented the social analysis of vehicular trace describing mobility in a large-scale metropolitan area. Using the realistic Zurich trace, we have characterized the mobility of vehicles according to macroscopic and microscopic social metrics. We showed the existence of similar behaviors and routines in the vehicles mobility. Furthermore, it was shown that the vehicles mobility could be characterized as a social network, following the laws of the degree distribution and the small distance between the nodes. It was also possible to verify that the use of social metrics can help in improving the performance of protocols, network infrastructure, and to propose new services to assist the drivers. As future works, we intend to extend the evaluation to traces with longer duration, aiming to analyze drives routine during different days of the week.

\section{REFERENCES}

[1] Azzedine Boukerche, Horacio A. B. F. Oliveira, Eduardo F. Nakamura, and Antonio A. F. Loureiro. Vehicular ad hoc networks: A new challenge for localization-based systems. Computer Communications, 31(12):28382849, jul 2008.

[2] P. Wagner C. Varschen. Mikroskopische modellierung der personenverkehrsnachfrage auf basis von zeitverwendungstagebchern. In Integrierte Mikro-Simulation von Raum- und Verkehrsentwicklung. Theorie, Konzepte, Modelle, Praxis, volume 81 of Stadt Region Land, pages 63-69, Berlin-Adlershof, 2006.

[3] Miad Faezipour, Mehrdad Nourani, Adnan Saeed, and Sateesh Addepalli. Progress and challenges in intelligent vehicle area networks. Communications ACM, 55(2):90-100, feb 2012.

[4] Marco Fiore and Jérôme Härri. The networking shape of vehicular mobility. In Proceedings of the 9th ACM international symposium on Mobile ad hoc networking and computing, MobiHoc '08, pages 261-272, New York, NY, USA, 2008. ACM.

[5] Xin Liu, Zhuo Li, Wenzhong Li, Sanglu Lu, Xiaoliang Wang, and Daoxu Chen. Exploring social properties in vehicular ad hoc networks. In Proceedings of the Fourth Asia-Pacific Symposium on Internetware, Internetware '12, pages 24:1-24:7, New York, NY, USA, 2012. ACM.

[6] Nicholas Loulloudes, George Pallis, and Marios D. Dikaiakos. The dynamics of vehicular networks in urban environments. CoRR, abs/1007.4106, 2010.

[7] Stanley Milgram. The small world problem. Psychology Today, 1(1):6167, 1967.

[8] Valery Naumov, Rainer Baumann, and Thomas Gross. An evaluation of inter-vehicle ad hoc networks based on realistic vehicular traces. In Proceedings of the 7th ACM international symposium on Mobile ad hoc networking and computing, MobiHoc '06, pages 108-119, New York, NY, USA, 2006. ACM.

[9] Yoann Pigné, Grégoire Danoy, and Pascal Bouvry. A vehicular mobility model based on real traffic counting data. In Proceedings of the Third international conference on Communication technologies for vehicles, Nets4Cars/Nets4Trains'11, pages 131-142, Berlin, Heidelberg, 2011. Springer-Verlag.

[10] Michal Piorkowski, Natasa Sarafijanovic-Djukic, and Matthias Grossglauser. CRAWDAD data set epfl/mobility (v. 2009-02-24). Downloaded from http://crawdad.cs.dartmouth.edu/epfl/mobility, February 2009.

[11] E. Schoch, F. Kargl, M. Weber, and T. Leinmuller. Communication patterns in vanets. Communications Magazine, IEEE, 46(11):119-125, 2008.

[12] S. J. T. University. Suvnet-trace data. Downloaded from http://wirelesslab.sjtu.edu.cn/download.html, 2007.

[13] Sandesh Uppoor and Marco Fiore. Insights on metropolitan-scale vehicular mobility from a networking perspective. In Proceedings of the 4th ACM international workshop on Hot topics in planet-scale measurement, HotPlanet '12, pages 39-44, New York, NY, USA, 2012. ACM.

[14] Duncan J. Watts and Steven H. Strogatz. Collective dynamics of 'smallworld' networks. Nature, 393(6684):440-442, June 1998. 